\title{
SP-DOMAINS ARE ALMOST DEDEKIND - A STREAMLINED PROOF
}

\author{
Malik Tusif Ahmed \\ Abdus Salam School of Mathematical Sciences \\ GC University, 68-B New Muslim Town \\ Lahore, Pakistan \\ e-mail: tusif.ahmad92@gmail.com \\ tusif.ahmed@sms.edu.pk
}

\begin{abstract}
Let $D$ be a domain. By [4], $D$ has "property SP" if every ideal of $D$ is a product of radical ideals. It is natural to consider property SP after studying Dedekind domains, which involve factoring ideals into prime ideals. In their article [4] Vaughan and Yeagy prove that a domain having property SP is an almost Dedekind domain. We give a very short and easy proof of this result.
\end{abstract}

Keywords: SP-domain, almost Dedekind domain, discrete valuation domain.

2010 Mathematics Subject Classification: Primary 13A15, Secondary $13 \mathrm{~F} 15$.

\section{REFERENCES}

[1] M.T. Ahmed and T. Dumitrescu, SP-rings with zero-divisors, Comm. Algebra 45 (3) (2017) 4435-4443. doi:10.1080/00927872.2016.1267184

[2] R. Gilmer, Multiplicative Ideal Theory, Queen's papers Pure Appl. Math. 90 (Queen's University, Kingston, Ontario, 1992).

[3] B. Olberding, Factorization into radical ideals, Arithmetical properties of commutative rings and monoids (S. Chapman, Ed.), Lecture Notes in Pure Appl. Math. 241 (Chapman \& Hall, 2005) 363-377.

doi:10.1201/9781420028249

[4] N.H. Vaughan, R.W. Yeagy, Factoring ideals into semiprime ideals, Canad. J. Math. XXX (6) (1978) 1313-1318.

doi:10.4153/CJM-1978-108-5

Received 29 August 2019

Revised 1 October 2019

Accepted 13 January 2020 